OPEN ACCESS

Edited by: James Cheng-Chung Wei, Chung Shan Medical University Hospital, Taiwan

Reviewed by: Ming-Shiou Jan, Chung Shan Medical University,

Taiwan

Chih-Wei Chen,

Chung Shan Medical

University, Taiwan

*Correspondence:

Oleg S. Glotor

olglotov@mail.ru

Specialty section: This article was submitted to Inflammation,

a section of the journa

Frontiers in Immunology

Received: 23 July 2021 Accepted: 11 October 2021 Published: 10 November 2021

Citation:

Shcherbak SG, Anisenkova AY Mosenko SV, Glotov OS, Chernov AN, Apalko SV, Urazov SP,

Garbuzov EY, Khobotnikov DN, Klitsenko OA, Minina EM and

Asaulenko ZP (2021) Basic Predictive Risk Factors for Cytokine Storms in COVID-19 Patients.

Front. Immunol. 12:745515. doi: 10.3389/fimmu.2021.745515

\section{Basic Predictive Risk Factors for Cytokine Storms in COVID-19 Patients}

\begin{abstract}
Sergey G. Shcherbak ${ }^{1,2}$, Anna Yu Anisenkova ${ }^{1,2}$, Sergei V. Mosenko ${ }^{1,2}$, Oleg S. Glotov ${ }^{1,3 *}$, Alexander N. Chernov ${ }^{1,4}$, Svetlana V. Apalko ${ }^{1}$, Stanislav P. Urazov ${ }^{1}$, Evgeny Y. Garbuzov ${ }^{1}$, Dmitry N. Khobotnikov ${ }^{1}$, Olga A. Klitsenko ${ }^{5}$, Evdokia M. Minina ${ }^{1}$ and Zakhar P. Asaulenko ${ }^{1,5}$

${ }^{1}$ City Hospital №40, Saint Petersburg, Russia, ${ }^{2}$ Department of Postgraduate Medical Education, Saint Petersburg State University, Saint Petersburg, Russia, ${ }^{3}$ Federal State-Financed Institution Pediatric Research and Clinical Center for Infectious Diseases under the Federal Medical Biological Agency, Saint Petersburg, Russia, ${ }^{4}$ Department of Pathphysiology, Institute of Experimental Medicine, Saint Petersburg, Russia, ${ }^{5}$ North-Western State Medical University named after Ivan Ivanovich (I.I.) Mechnikov, Saint Petersburg, Russia
\end{abstract}

Objective: A critical role in coronavirus disease 2019 (COVID-19) pathogenesis is played by immune dysregulation that leads to a generalized uncontrolled multisystem inflammatory response, caused by overproduction of proinflammatory cytokines, known as "a cytokine storm" (CS), strongly associated with a severe course of disease. The aim of this study is to identify prognostic biomarkers for CS development in COVID-19 patients and integrate them into a prognostic score for CS-associated risk applicable to routine clinical practice.

Materials and Methods: The authors performed a review of 458 medical records from COVID-19 patients (241 men and 217 women aged $60.0 \pm 10.0$ ) who received treatment in the St. Petersburg State Budgetary Institution of Healthcare City Hospital 40 (City Hospital 40, St. Petersburg), from Apr. 18, 2020 to Nov. 21, 2020. The patients were split in two groups: one group included 100 patients with moderate disease symptoms; the other group included 358 patients with progressive moderately severe, severe, and extremely severe disease. The National Early Warning Score (NEWS) score was used alongside with clinical assessment, chest computed tomographic (CT) scans, electrocardiography (ECG), and lab tests, like ferritin, C-reactive protein (CRP), interleukin (IL)-6, lactate dehydrogenase (LDH), and D-dimer.

Results: The basic risk factors for cytokine storms in COVID-19 patients are male gender, age over 40 years, positive test result for replicative severe acute respiratory syndrome coronavirus 2 (SARS-CoV-2) RNA, absolute lymphocyte count, dynamics in the NEWS score, as well as LDH, D-dimer, ferritin, and IL-6 levels. These clinical and instrumental findings can be also used as laboratory biomarkers for diagnosis and dynamic monitoring of cytokine storms. The suggested prognostic scale (including the NEWS score dynamics; 
serum IL-6 greater than $23 \mathrm{pg} / \mathrm{ml}$; serum CRP $50 \mathrm{mg} / \mathrm{L}$ or greater; absolute lymphocyte count less than $0.72 \times 10^{9} / \mathrm{L}$; positive test result for replicative coronavirus (SARS-CoV-2) RNA; age 40 years and over) is a useful tool to identify patients at a high risk for cytokine storm, requiring an early onset of anti-inflammatory therapy.

Keywords: COVID-19, cytokine storm, clinical and biochemical prognostic factors, patients, prognostic scale

\section{INTRODUCTION}

Coronavirus disease 2019 (COVID-19) infection caused by SARS-CoV-2 coronavirus remains a global challenge for the world's healthcare system. Most people infected with SARSCoV-2 will experience mild illness. Some patients, however, develop a dysregulated immune response, inducing severe lung injury. This is manifest as acute respiratory distress syndrome (ARDS) which precedes acute respiratory failure, extrapulmonary organ dysfunction, and high risk of death. COVID-19 disease is usually associated with elevated inflammatory biomarkers, cytokines, and chemokines, especially in severe cases. Moreover, lymphocytopenia and neutrophilia often emerged, with particularly declined counts of CD8+ T cells, CD4+ T cells, and natural killer (NK) cells (1). The inpatient death rate varies between $15 \%$ and $20 \%$ or even higher among those requiring ICU admission (2). A critical role in COVID-19 pathogenesis is played by immune dysregulation that leads to a generalized uncontrolled multisystem inflammatory response, caused by overproduction of proinflammatory cytokines, known as "a cytokine storm" (CS). The CS manifestations are fever, cytopenia, hyperferritinemia, abnormal levels of liver transaminases, coagulopathy, and lung damage (including ARDS) (3). In all these conditions, cytokines interleukin (IL)-1 $\beta$, IL-18, IFN- $\gamma$, and IL- 6 are the key mediators responsible for the hyperinflammatory state. COVID-19-associated CS is a unique form of hyperinflammatory response, requiring further characterization criteria (4). The aim of this study is to identify prognostic biomarkers for CS development in COVID-19 patients and integrate them into a prognostic score for CSassociated risk applicable to routine clinical practice.

\section{MATERIALS AND METHODS}

\section{Study Design and Inclusion Criteria}

The study design is an observational clinical trial. We utilized 458 medical records from COVID-19 patients who received treatment in the St. Petersburg State Budgetary Institution of Healthcare City Hospital 40 (City Hospital 40, St. Petersburg) from Apr. 18, 2020 to Nov. 21, 2020; the patients tested positive for SARS-CoV-2 RNA by polymerase chain reaction (PCR) amplification of nucleic acids from clinical material and presented clinical manifestations and symptoms (fever, general fatigue, malaise, cough, and dyspnea), features of viral pneumonia seen on unenhanced lung CT scan (noted as multiple lobular abnormalities often located in the peripheral areas of the lower lobes and manifested with predominantly perivascular bilateral disease distribution; multiple peripheral areas of ground-glass opacities with rounded morphology and variable extent; interlobular septal thickening/flattening that causes a crazy-paving pattern, areas of consolidation, air bronchogram sign, etc.) $(5,6)$.

\section{Characteristics of Groups of Patients}

Table 1 shows demographic characteristics, history, and comorbidities of the patient study cohort.

In accordance with the International and Russian Recommendations for the Prevention, Diagnosis and Treatment of New Coronavirus Infection (COVID-19), all patients were divided in two groups of comparable age $(7,8)$. The first group included $100(21.8 \%)$ patients with clinical and radiological characteristics of stable, moderately severe course of disease. The criteria for an easy course were considered to be body temperature below $38^{\circ} \mathrm{C}$, cough, weakness, sore throat, and the absence of criteria for moderate and severe course. The criteria for a moderate course are fever, temperature above $38^{\circ} \mathrm{C}$, respiratory rate over $22 / \mathrm{min}$, shortness of breath during exercise, pneumonia (exposed to CT of the lungs), and $\mathrm{SpO}_{2}$ $<95 \%$. The second group included $358(78.2 \%)$ patients with progressive, moderately severe, severe, and extremely severe course of disease. Clinical and radiological criteria for severe course were respiratory rate more than $30 / \mathrm{min}, \mathrm{SpO}_{2} 93 \%, \mathrm{PaO}_{2} /$ $\mathrm{FiO}_{2} 300 \mathrm{mmHg}$, progression of changes in the lungs typical for COVID-19 pneumonia according to CT data, including an increase in the prevalence of revealed changes by more than $25 \%$, as well as the appearance of signs of other pathological conditions, changes in the level of consciousness, unstable hemodynamics (systolic blood pressure less than $90 \mathrm{mmHg}$ or diastolic blood pressure less than $60 \mathrm{mmHg}$, urine output less than $20 \mathrm{ml} / \mathrm{h}$ ), and $\mathrm{qSOFA}>2$ points. The criteria for an extremely severe course were signs of ARF with the need for respiratory support (invasive ventilation), septic shock, and multiple organ failure. Thus, patients with mild and moderate and severe and extremely severe course are shown in Table 2.

Symptoms at admission were fever in 365 (80\%) patients, cough in 329 (72\%) patients, dyspnea in 265 (57.86\%) patients, myalgia in $43(9.39 \%)$ patients, general fatigue in $344(75.11 \%)$ patients, headache in $36(7.86 \%)$ patients, a sore throat in 29 $(6.33 \%)$ patients, a runny nose or rhinorrhea in $46(10.04 \%)$ patients, chest pain in $51(11.14 \%)$ patients, diarrhea in 34 (7.42\%) patients, nausea and vomiting in 13 (2.84\%) patients, and impaired taste and smell in 40 (8.73\%) patients. Four hundred fifty $(98.25 \%)$ reported one or more disease-specific 
TABLE 1 | Demographic characteristics, history, and comorbidities of the patient study cohort.

\begin{tabular}{lc}
\hline Parameter & $n$ (\%) \\
\hline Age (years) & $38(8.30 \%)$ \\
$\leq 39$ & $58(12.66 \%)$ \\
$40-49$ & $123(26.86 \%)$ \\
$50-59$ & $139(30.35 \%)$ \\
$60-69$ & $100(21.83 \%)$ \\
$\geq 70$ & $100(22.22 \%)$ \\
History of exposure to infected individuals & $45(9.83 \%)$ \\
History of travel outside the place of residence during the past 14 days & $44(9.61 \%)$ \\
Flu-resembling in relatives, including fever, cough, fatigue & $260(56.77 \%)$ \\
History of other diseases & $222(48.47 \%)$ \\
Hypertension & $139(30.35 \%)$ \\
Coronary artery disease & $97(21.18 \%)$ \\
Cerebrovascular disease & $34(7.42 \%)$ \\
Poststroke condition & $89(19.43 \%)$ \\
Condition following acute myocardial infarction & $65(14.19 \%)$ \\
Condition following a surgical intervention & $63(13.76 \%)$ \\
Rheumatoid arthritis and other autoimmune diseases & $32(6.99 \%)$ \\
Diabetes mellitus & $22(4.80 \%)$ \\
Chronic kidney disease, stages 3 to 5 & $20(4.37 \%)$ \\
Malignant lesions & $20(4.37 \%)$ \\
Chronic obstructive pulmonary disease & $13(2.84 \%)$ \\
Chronic bronchitis & \\
Chronic asthma &
\end{tabular}

symptoms. CT imaging manifestation of pneumonia was detected in $458(100 \%)$ patients.

\section{Clinical and Biochemical Surveillances}

Medical examination of all patients included history taking, with a focus on the course of disease, physical examination, and assessment of hemodynamics and respiratory system (respiratory rate, heart rate, blood pressure, $\mathrm{SpO}_{2}$, respiratory distress); calculation of the National Early Warning Score (NEWS), a recommended scoring system for use in COVID-19 patients (10, $11)$; computed tomography (CT) of the chest with the severity score ranking on a 4-point scale (CT-1, CT-2, CT-3, CT-4); laboratory tests (complete haemogram, basic blood chemistry panel, ferritin test, C-reactive protein, IL-6, lactate dehydrogenase test, D-dimer); ECG; and other instrumental examinations, if required.

\section{Therapy for Patients With COVID-19 Infection}

In group 1, treatment of COVID-19 and its complications included antibacterial and antiviral drugs, prevention of hypercoagulability and disseminated intravascular coagulation, symptom-related treatment, and oxygen therapy. To prevent or treat the cytokine storm depending on the disease severity, in group 2, standard treatment was supplemented with convalescent plasma therapy, anticytokine drugs: interleukine-6 (IL-6) inhibitors (tocilizumab, olokizumab, levilimab), IL-1

TABLE 2 | Disease severity in different groups of patients.

\begin{tabular}{|c|c|c|c|c|c|c|}
\hline \multirow[t]{2}{*}{ Parameter } & \multicolumn{2}{|c|}{ Group 1} & \multicolumn{2}{|c|}{ Group 2} & \multirow[t]{2}{*}{ Total } & \multirow[t]{2}{*}{$p$-value } \\
\hline & $n$ & $\%$ & $n$ & $\%$ & & \\
\hline Males & 58 & 58.0 & 159 & 44.4 & 217 & 0.016 \\
\hline Females & 42 & 42.0 & 199 & 55.6 & 241 & \\
\hline \multicolumn{7}{|l|}{ Moderate severity of disease } \\
\hline Moderate & 100 & 100.00 & 153 & 42.74 & 253 & 0.000 \\
\hline Severe and extremely severe & 0 & 0.00 & 205 & 57.26 & 205 & \\
\hline \multicolumn{7}{|c|}{ Lung involvement evaluation at admission based on a 4-point CT score ranking } \\
\hline $\mathrm{CT}-1$ & 57 & 57.0 & 82 & 22.9 & 139 & \\
\hline CT-2 & 43 & 43.0 & 223 & 62.3 & 263 & 0.000 \\
\hline CT-3 & 0 & 0.0 & 44 & 12.3 & 47 & \\
\hline CT-4 & 0 & 0.0 & 9 & 2.5 & 9 & \\
\hline \multicolumn{7}{|l|}{ Outcomes } \\
\hline Survivals & 100 & 100.0 & 255 & 71.2 & 355 & 0.000 \\
\hline Deaths & 0 & 0.0 & 103 & 28.8 & 103 & \\
\hline
\end{tabular}

Assessment of the severity of changes in the lungs (volume, area, length) in patients with COVID-19 pneumonia was carried out visually on the basis of computer programs for assessing the volume of compacted lung tissue and mapping the density of the pulmonary parenchyma in both lungs: (1) Absence of characteristic manifestations (CT-0). (2) Minimum volume/ prevalence $<25 \%$ of lung volume (CT-1). (3) Average volume/prevalence $25 \%-50 \%$ of lung volume (CT-2). (4) Significant volume/prevalence of $50 \%-75 \%$ of lung volume (CT-3). (5) Critical volume prevalence $>75 \%$ of lung volume (CT-4) (9). 
inhibitors (canakinumab, RH104), JAK inhibitors (tofacitinib, ruxolitinib, baricitinib), Bcr-Abl tyrosine kinase inhibitor (radotinib), and glucocorticoids for some cases. Respiratory therapy, modified antibacterial therapy, extracorporeal membrane oxygenation, sepsis, and septic shock treatment (extracorporeal detoxication and blood purification, etc.) were performed in a stepwise fashion according to indications (5).

\section{Statistical Analysis}

Data were analyzed using STATISTICA for Windows (version 10, license No. BXXR310F964808FA-V) software. Since all data (except age) did not follow a normal distribution, we applied the Mann-Whitney, Kolmogorov-Smirnov, median Chi-square, and ANOVA tests to evaluate between-group differences in quantitative parameters (age, NEWS score, D-dimer, C-reactive protein (CRP), IL-6, etc.) and normality of sampling distribution. We used Pearson's Chi-squared and Fischer's exact nonparametric tests to assess the frequencies of qualitative data (gender, disease manifestations and severity, complaints). Classification Trees were utilized to identify cutoff thresholds for age, NEWS score, and lab test values. The odds ratio (OR) for developing the CS-the ratio of probabilities of a certain event for different groups-was calculated for a $2 \times 2$ contingency table using a standard formula and the associated confidence interval. We applied the Haldane's correction, if the $2 \times 2$ table contained a zero cell.

\section{RESULTS}

\section{Comparison of Clinical and Lab Variables in Different Groups of Patients}

At admission, group 1 patients showed a reliably higher frequency of chest CT score 1, whereas group 2 patients were admitted with more extensive lung involvement (CT score ranging from 2 to 4). Although moderately severe lung involvement (CT score 2) prevailed in group 2, at admission, these patients reported manifestations of progressive respiratory failure and fever (Table 3). There is a reliable difference in the NEWS score: group 1 had the average admission NEWS score of 2 and the average hospital stay of 11 days; group 2 had the average NEWS score of 4 at admission which exacerbated to 5 at the start of treatment, including anticytokine drugs, anti-COVID-19 convalescent plasma, and hemoadsorbtion; the average hospital stay in group 2 was 12 days. The death rate due to comorbidities was highest in group 2 patients with severe and extremely severe course of disease (28.8\% in group 2 and $22.5 \%$ in the total cohort). At baseline, such patients had an unfavorable forecast due to age, comorbidity, clinical severity of acute respiratory failure, a high NEWS score, dynamic evaluation of extensive lung involvement, and exacerbation of lung injury based on chest CT (Table 3). The virus-induced infection elicits inflammatory manifestations (absolute lymphocyte count and levels of LDG, CRP, ferritin, D-dimer, and IL-6) that correlate with a CS scenario (lymphocytopenia, hypercytokinemia, hyperinflammation) (12).

In addition, prevalence of comorbidities in our cohort was greater than reported by other authors for adult patients with
COVID-19 (31\%) (13). The high rate of comorbidities in our patients is due to the specific profile of our department, which focuses on patients with a severe and extremely severe course of disease. Two hundred twenty-one (48\%) patients were admitted to ICU from other departments or hospitals due to COVID19 progression.

\section{Bioinformatic Analysis of Patient Data and Critical CS-Related Clinical, Instrumental, and Lab Characteristics}

Comparative analysis of clinical, imaging, and lab characteristics in recruited cohorts of patients has revealed most critical CSrelated variables (Table 4 ).

The NEWS score dynamics across different patient groups exhibits qualitative differences: the NEWS score decreased from baseline in group 1 (by $-1[-2 ; 0]$ points) and increased in group 2 with progressive course of disease (by $+1[0 ; 2]$ points) $(p<$ $0.001)$. There are reliable between-group differences in lab variables (absolute lymphocyte count, CRP, ferritin, D-dimer, IL-6), which correlate with the NEWS score dynamics at admission until the start of CS treatment.

Classification Trees were utilized to identify cutoffs for CSassociated risk factors (Table 5).

Group 2 patients showed a reliably higher frequency of prognostic variables exceeding CS-associated cutoff values (Table 6).

This was followed by a comprehensive evaluation of CSassociated risk based on the ranking of variables obtained at admission. These variables were ranked by prognostic relevance in accordance with Classification Trees by using CART-style method for Split selection and included the following: NEWS score dynamics, serum IL-6 greater than $23 \mathrm{pg} / \mathrm{ml}$, serum CRP $50 \mathrm{mg} / \mathrm{l}$ and greater, absolute lymphocyte count less than $0.72 \times$ $10^{9} / \mathrm{L}$, positive test for replicative SARS-CoV-2 RNA, and age 40 years and over. An assessment of the effectiveness of the obtained criteria was carried out, which confirmed the possibility of their application in clinical practice. These biomarkers can be used as predictive criteria to determine the CS risk. We should note that there are no meaningful gender-related differences in the comprehensive examination of CS-related risk.

Figure 1 shows incremental CS-associated risk (OR) and its correlation with lab test values.

Increased CS incidence is associated with a larger number of risk factors (correlation coefficient $\mathrm{Rg}=+0.91, p<0.001$ ) (Table 6; Figure 2). Each of these factors, if combined with the largest number of other risk factors, exacerbated the CSassociated risk.

We outlined the following risk categories of patients to enhance clinical relevance of our prognostic model: category 1 ( 0 to 1 risk factors $)=\mathrm{CS}$-associated risk is near zero; category 2 (two to three risk factors) $=\mathrm{CS}$-associated risk escalates dramatically to $55 \%$, augmenting 35.5 -fold vs. category 1; category 3 (four and more risk factors) = CS-associated risk exacerbates to $96 \%$, augmenting 718 -fold vs. category 1 . The obtained results are consistent with findings by other authors on the evaluation of risk factors for COVID-19-associated CS (14, 
TABLE 3 | Between-group differences in NEWS score, time from symptoms onset to hospital admission, and length of hospital stay.

\begin{tabular}{|c|c|c|c|c|c|c|}
\hline \multirow[t]{2}{*}{ Parameter } & \multirow[t]{2}{*}{ Characteristics } & \multicolumn{2}{|c|}{ Group 1} & \multicolumn{2}{|r|}{ Group 2} & \multirow[t]{2}{*}{$p$-value } \\
\hline & & $n$ & Value & $n$ & Value & \\
\hline NEWS score at admission & $\begin{array}{c}\mathrm{M} \pm \mathrm{SD} \\
\min \div \max \end{array}$ & 100 & $\begin{array}{c}2.4 \pm 1.7 \\
0 \div 8\end{array}$ & 356 & $\begin{array}{c}4.5 \pm 2.7 \\
0 \div 14\end{array}$ & $<0.001$ \\
\hline NEWS score by the start of CS treatment & $\begin{array}{c}\mathrm{M} \pm \mathrm{SD} \\
\min \div \max \end{array}$ & 100 & $\begin{array}{c}1.5 \pm 1.6 \\
0 \div 6\end{array}$ & 357 & $\begin{array}{c}5.68 \pm 2.82 \\
0 \div 14\end{array}$ & $<0.001$ \\
\hline NEWS score at discharge & $\begin{array}{c}\mathrm{M} \pm \mathrm{SD} \\
\min \div \max \end{array}$ & 100 & $\begin{array}{c}0.2 \pm 1.02 \\
0 \div 9\end{array}$ & 349 & $\begin{array}{c}3.29 \pm 5.42 \\
0 \div 16\end{array}$ & $<0.001$ \\
\hline Days from symptoms onset to hospital admission & $\begin{array}{c}\mathrm{M} \pm \mathrm{SD} \\
\min \div \max \end{array}$ & 100 & $\begin{array}{c}8.8 \pm 5.9 \\
0 \div 37\end{array}$ & 356 & $\begin{array}{c}6.63 \pm 5.39 \\
0 \div 57\end{array}$ & $<0.001$ \\
\hline $\begin{array}{l}\text { Day of disease on which CS treatment started (anticytokine drugs, convalescent plasma, } \\
\text { hemoadsorbtion) }\end{array}$ & $\begin{array}{c}\mathrm{M} \pm \mathrm{SD} \\
\min \div \max \end{array}$ & 100 & $\begin{array}{c}9.0 \pm 6.0 \\
1 \div 37\end{array}$ & 357 & $\begin{aligned} 10.35 & \pm 5.98 \\
1 & \div 59\end{aligned}$ & $<0.017$ \\
\hline Length of hospital stay (days in hospital) & $\mathrm{M} \pm \mathrm{SD}$ & 100 & $11.8 \pm 4.9$ & 355 & $13.6 \pm 6.7$ & $<0.012$ \\
\hline
\end{tabular}

The NEWS2 is based on a simple aggregate scoring system in which a score is allocated to six physiological measurements (respiration rate, oxygen saturation, systolic blood pressure, pulse rate, level of consciousness) when patients present to, or are being monitored in hospital.

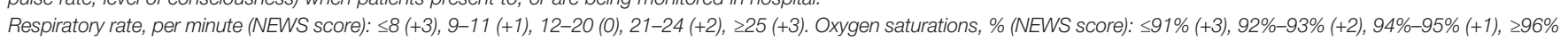
(0). Temperature, ${ }^{\circ} \mathrm{C} /{ }^{\circ} \mathrm{F}$ (NEWS score): $\leq 35^{\circ} \mathrm{C} / 95^{\circ} \mathrm{F}(+3), 35.1^{\circ}-36^{\circ} \mathrm{C} / 95.1^{\circ} \mathrm{F}-96.8^{\circ} \mathrm{F}(+1), 36.1^{\circ} \mathrm{C}-38^{\circ} \mathrm{C} / 96.9^{\circ} \mathrm{F}-100.4^{\circ} \mathrm{F}(0), 38.1^{\circ} \mathrm{C}-39^{\circ} \mathrm{C} / 100.5^{\circ} \mathrm{F}-102.2^{\circ} \mathrm{F}(+1), \geq 39.1^{\circ} \mathrm{C} / 102.3^{\circ} \mathrm{F}(+2)$. Systolic blood pressure, $\mathrm{mmHg}$ (NEWS score): $\leq 90$ (+3), 91-100 (+2), 101-110 (+1), 111-219 (0), $\geq 220$ (+3).

Pulse rate, per minute (NEWS score): $\leq 40$ (+3), 41-50 (+1), 51-90 (0), 91-110 (+1), 111-130) (+2). $\geq 131(+3)$;

Consciousness: (NEWS score): alert (0), voice, pain, unresponsive (+3) (11).

15). Our results provide a justification for a treatment strategy with an early onset of proactive anti-inflammatory therapy and anti-COVID-19 convalescent plasma in patients at a high risk for CS development.

\section{DISCUSSION}

Considering there are no reliable prognostic criteria for COVID19-induced CS available today, we selected 458 patients with a different course of disease to evaluate prognostic power of available clinical, instrumental, and lab parameters; the most useful parameters for assessing outcome were collated into coherent domains, or clusters, and their prognostic power was evaluated. The clinical data collected from patient histories and at hospital admission included signs and symptoms, demographic, epidemiological, and clinical parameters, assessment of disease severity using the NEWS score, severity of COVID-19, comorbidities, dynamic evaluation of lung involvement (groundglass opacities \pm areas of consolidation) based on a standard imaging protocol for unenhanced chest CT (5), serum lab test values (16) recorded within $24 \mathrm{~h}$ prior or after the diagnosis of CS and over the next 7 days of hospital stay. Test results for replicative SARS-CoV-2 RNA, length of hospital stay and outcomes were assessed over the next 10 days. Potential risk factors for CS development were identified by comparing between patients with and without CS-associated clinical and CT manifestations. A deteriorating NEWS score is associated with a clinically severe disease and progressive hemodynamic impairment. Thus, in group 1 patients, admission NEWS score was no greater than 4 , improving by 1-2 points after the onset of treatment; in contrast,

TABLE 4 | Comparative analysis of basic variables for CS diagnosis at the onset of proactive anti-inflammatory treatment.

\begin{tabular}{|c|c|c|c|c|c|}
\hline \multirow[t]{2}{*}{ Parameter } & \multicolumn{2}{|c|}{ Group 1} & \multicolumn{2}{|c|}{ Group 2} & \multirow[t]{2}{*}{$p$-value } \\
\hline & $n$ & $M \pm S D \min \div \max$ & $n$ & $M \pm S D \min \div \max$ & \\
\hline Age (year) & 100 & $\begin{array}{c}57.53 \pm 15.06 \\
21 \div 86\end{array}$ & 358 & $\begin{array}{c}60.5 \pm 13.37 \\
24 \div 89\end{array}$ & 0.05 \\
\hline Serum lymphocytes $\left(10^{9} / \mathrm{L}\right)$ & 98 & $\begin{array}{l}1.49 \pm 0.59 \\
0.46 \div 3.2\end{array}$ & 349 & $\begin{array}{l}1.28 \pm 1.39 \\
0.23 \div 24.62\end{array}$ & $<0.01$ \\
\hline Serum LDH (U/L) & 27 & $\begin{array}{c}357.78 \pm 155.3 \\
169 \div 914\end{array}$ & 149 & $\begin{array}{c}410.17 \pm 191.24 \\
134 \div 1492\end{array}$ & $<0.1$ \\
\hline Serum CRP (mg/L) & 91 & $\begin{array}{r}54.61 \pm 64.92 \\
0.5 \div 274.9\end{array}$ & 346 & $\begin{array}{r}106.71 \pm 79.58 \\
0.8 \div 361.9\end{array}$ & $<0.001$ \\
\hline Serum ferritin (ng/ml) & 20 & $\begin{array}{c}328.57 \pm 185.15 \\
57.1 \div 781.3\end{array}$ & 190 & $\begin{array}{r}696.28 \pm 792.88 \\
0 \div 7759.4\end{array}$ & $<0.01$ \\
\hline Serum D-dimer ( $\mu \mathrm{g} / \mathrm{ml})$ & 29 & $\begin{array}{l}1.26 \pm 2.75 \\
0.27 \div 15.34\end{array}$ & 147 & $\begin{array}{l}1.84 \pm 2.79 \\
0.15 \div 18.69\end{array}$ & $<0.05$ \\
\hline Serum IL-6 (pg/ml) & 65 & $\begin{array}{r}15.02 \pm 23.64 \\
0 \div 127.2\end{array}$ & 318 & $\begin{aligned} 161.26 & \pm 442.5 \\
1.5 & \div 4894\end{aligned}$ & $<0.001$ \\
\hline NEWS score dynamics from admission to the onset of CS treatment & 100 & $\begin{aligned}-0.96 & \pm 1.19 \\
-4 & \div 4\end{aligned}$ & 356 & $\begin{array}{c}1.24 \pm 1.86 \\
-3 \div 11\end{array}$ & $<0.001$ \\
\hline
\end{tabular}


TABLE 5 | Cutoffs for predictive risk factors of CS in groups 1 and 2 at the onset of proactive anti-inflammatory treatment.

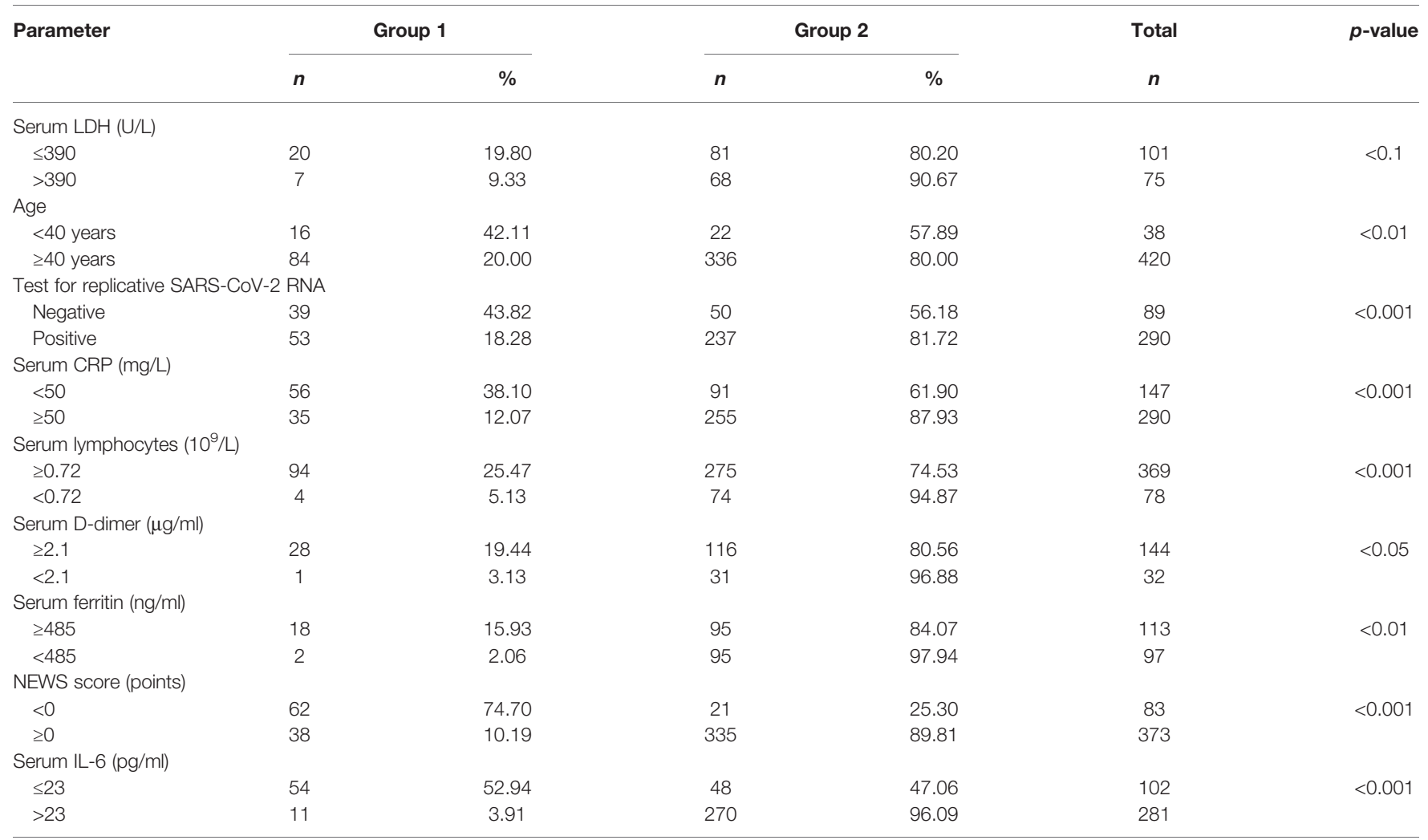

The table shows the threshold values for all parameters that are highlighted as borderline for the risk of developing a cytokine storm.

TABLE 6 | CS incidence rate depending on the number of risk factors.

\begin{tabular}{|c|c|c|c|c|c|}
\hline No. of CS-associated risk factors & \multicolumn{2}{|c|}{ Group 1} & \multicolumn{2}{|c|}{ Group 2} & Total \\
\hline None & 2 & 100.00 & 0 & 0.00 & 2 \\
\hline 2 factors & 14 & 63.64 & 8 & 36.36 & 22 \\
\hline 3 factors & 21 & 37.50 & 35 & 62.50 & 56 \\
\hline 4 factors & 6 & 9.68 & 56 & 90.32 & 62 \\
\hline Total & 57 & 18.39 & 253 & 81.61 & 310 \\
\hline
\end{tabular}
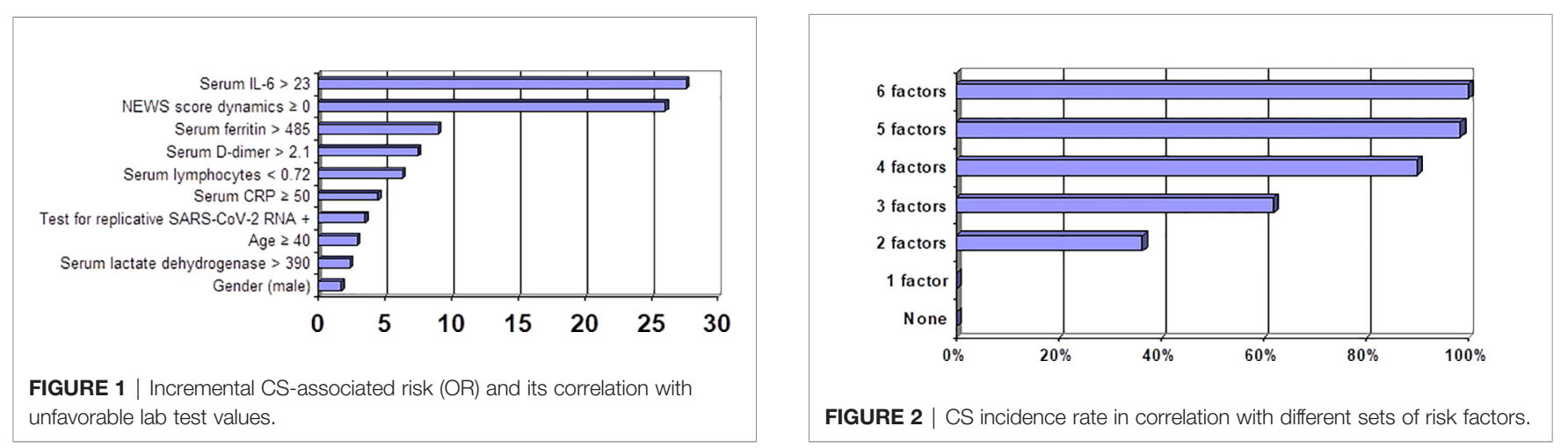
the NEWS score was higher in group 2 patients at baseline, exacerbating further by $1.24 \pm 1.86$ points. Groups 1 and 2 presented reliable differences in IL-6, CRP, ferritin, and lymphocyte count values. In conclusion, our findings show that a progressive course of disease is associated with escalating values of biomarkers that induced the CS-associated clinical scenario in recruited patients.

\section{CONCLUSIONS}

1. The basic risk factors for cytokine storms in COVID-19 patients include male gender, lactate dehydrogenase level, age over 40 years, positive test result for replicative SARSCoV-2 RNA, absolute lymphocyte count, D-dimer and ferritin levels, dynamics in the NEWS score, and plasma IL-6 concentration.

2. Absolute lymphocyte count, LDH, CRP, ferritin, D-dimer, and IL-6 levels are the most critical lab parameters for diagnosis and dynamic monitoring of cytokine storms.

3. The suggested prognostic scale (including the NEWS score dynamics; serum IL-6 greater than $23 \mathrm{pg} / \mathrm{ml}$; serum CRP = $50 \mathrm{mg} / \mathrm{L}$ or greater; absolute lymphocyte count less than 0.72 $\times 10^{9} / \mathrm{L}$; positive test result for replicative coronavirus (SARS-CoV-2) RNA; age 40 years and over) is a useful tool to identify patients at a high risk for cytokine storm, requiring an early onset of anti-inflammatory therapy.

\section{DATA AVAILABILITY STATEMENT}

The original contributions presented in the study are included in the article/Supplementary Material. Further inquiries can be directed to the corresponding author.

\section{REFERENCES}

1. Chen G, Wu D, Guo W, Cao Y, Huang D, Wang H, et al. Clinical and Immunological Features of Severe and Moderate Coronavirus Disease 2019. J Clin Invest (2020) 130(5):2620-9. doi: 10.1172/JCI137244

2. Wiersinga WJ, Rhodes A, Cheng AC, Peacock SJ, Prescott HC. Pathophysiology, Transmission, Diagnosis, and Treatment of Coronavirus Disease 2019 (COVID19): A Review. JAMA (2020) 324(8):782-93. doi: 10.1016/j.jiph.2020.09.008

3. Caso F, Costa L, Ruscitti P, Navarini L, Del Puente A, Giacomelli R, et al. Could Sars-Coronavirus-2 Trigger Autoimmune and/or Autoinflammatory Mechanisms in Genetically Predisposed Subjects? Autoimmun Rev (2020) 19 (5):102524. doi: 10.1016/j.autrev.2020.102524

4. Zachariah P, Johnson CL, Halabi KC, Ahn D, Sen AI, Fischer A, et al. Epidemiology, Clinical Features, and Disease Severity in Patients With Coronavirus Disease 2019 (COVID-19) in a Children's Hospital in New York City, New York. JAMA Pediatr (2020) 174(10):e202430. doi: 10.1001/jamapediatrics.2020.2430

5. Belfiore MP, Urraro F, Grassi R, Giacobbe G, Patelli G, Cappabianca S, et al. Artificial Intelligence to Codify Lung CT in Covid-19 Patients. Radiol Med (2020) 125(5):500-4. doi: 10.1007/s11547-020-01195-X

6. Fang Y, Zhang H, Xie J, Lin M, Ying L, Pang P, et al. Sensitivity of Chest CT for COVID-19: Comparison to RT-PCR. Radiology (2020) 296(2):E115-7. doi: $10.1148 /$ radiol.2020200432

\section{ETHICS STATEMENT}

The studies involving human participants were reviewed and approved by City Hospital No. 40, Saint Petersburg. The patients/participants provided their written informed consent to participate in this study.

\section{AUTHOR CONTRIBUTIONS}

SS: concept development and research; guidance, choice of treatment strategy, and patient assessment; and analysis of the studied groups. AA, EG, and DK: treatment and examination of patients. SM and SU: literary search. AA and SM: formation of the base of the studied groups, statistical analysis, and writing an article. OK: statistical analysis. EM, ZA, and OG: performing $\mathrm{X}$-ray, pathomorphological diagnostics and testing biological samples of patients for the presence of SARS-CoV-2 coronavirus. OG and AC: editorial of the article. SA: biobanking of materials. All authors contributed to the article and approved the submitted version.

\section{FUNDING}

The research was financed from the budget of the St. Petersburg State Budgetary Institution of Healthcare City Hospital 40 and a grant from the funds of St. Petersburg State University ID PURE: 75253103.

\section{SUPPLEMENTARY MATERIAL}

The Supplementary Material for this article can be found online at: https://www.frontiersin.org/articles/10.3389/fimmu.2021. 745515/full\#supplementary-material

7. WHO. Clinical Management of COVID-19. Interium Guidance (Version 05/ 20/2020). Geneva: WHO (2020).

8. Interim guidelines. Prevention, Diagnosis and Treatment of New Coronavirus Infection (COVID-19). Version 12 (09/21/2021) Approved by the Ministry of Health of the Russian Federation.

9. Inui S, Fujikawa A, Jitsu M, Kunishima N, Watanabe S, Suzuki Y, et al. Chest CT Findings in Cases From the Cruise Ship Diamond Princess With Coronavirus Disease (COVID-19). Radiol Cardiothorac Imaging (2020) 2 (2):e200110. doi: 10.1148/ryct.2020200110

10. Royal College of Physicians. National Early Warning Score (NEWS) 2: Standardising the Assessment of Acute-Illness Severity in the NHS. Updated Report of a Working Party. London: RCP (2017).

11. Carr E, Bendayan R, Bean D, Stammers M, Wang W, Zhang H, et al. Evaluation and Improvement of the National Early Warning Score (NEWS2) for COVID19: A Multi-Hospital Study. BMC Med (2021) 19:23. doi: 10.1186/s12916-02001893-3

12. Kivela P. Paradigm Shift for COVID-19 Response: Identifying High-Risk Individuals and Treating Inflammation. West J Emerg Med (2020) 21(3):4736. doi: 10.5811/westjem.2020.3.47520

13. Jutzeler CR, Bourguignon L, Weis CV, Tong B, Wong C, Riecka B, et al. Comorbidities, Clinical Signs and Symptoms, Laboratory Findings, Imaging Features, Treatment Strategies, and Outcomes in Adult and Pediatric Patients 
With COVID-19: A Systematic Review and Meta-Analysis. Travel Med Infect Dis (2020) 37:101825. doi: 10.1016/j.tmaid.2020.101825

14. Caricchio R, Gallucci M, Dass C, Zhang X, Gallucci S, Fleeceet D, et al. Preliminary Predictive Criteria for COVID-19 Cytokine Storm. Ann Rheum Dis (2021) 80(1):88-95. doi: 10.1136/annrheumdis-2020-218323

15. Moore J, June C. Cytokine Release Syndrome in Severe COVID-19. Science (2020) 368(6490):473-4. doi: 10.1126/science.abb8925

16. Lippi G, Plebani M. Laboratory Abnormalities in Patients With COVID2019 Infection. Clin Chem Lab Med (2020) 58(7):1131-4. doi: 10.1515/cclm2020-0198

Conflict of Interest: The authors declare that the research was conducted in the absence of any commercial or financial relationships that could be construed as a potential conflict of interest.
Publisher's Note: All claims expressed in this article are solely those of the authors and do not necessarily represent those of their affiliated organizations, or those of the publisher, the editors and the reviewers. Any product that may be evaluated in this article, or claim that may be made by its manufacturer, is not guaranteed or endorsed by the publisher.

Copyright (c) 2021 Shcherbak, Anisenkova, Mosenko, Glotov, Chernov, Apalko, Urazov, Garbuzov, Khobotnikov, Klitsenko, Minina and Asaulenko. This is an open-access article distributed under the terms of the Creative Commons Attribution License (CC BY). The use, distribution or reproduction in other forums is permitted, provided the original author(s) and the copyright owner(s) are credited and that the original publication in this journal is cited, in accordance with accepted academic practice. No use, distribution or reproduction is permitted which does not comply with these terms. 